\title{
Quand le numérique s'invite au château : les serious games comme outil de médiation $d u$ patrimoine
}

When digital technology storms the castle: serious games as a tool reflexion on heritage

\section{Catherine Bouko}

\section{CpenEdition}

Journals

Édition électronique

URL : http://journals.openedition.org/edc/6465

DOI : 10.4000/edc.6465

ISSN : 2101-0366

Éditeur

Université Lille-3

\section{Édition imprimée}

Date de publication : 1 décembre 2015

Pagination : 97-112

ISBN : 978-2-917562-14-7

ISSN : $1270-6841$

\section{Référence électronique}

Catherine Bouko, «Quand le numérique s'invite au château : les serious games comme outil de médiation du patrimoine », Études de communication [En ligne], 45 | 2015, mis en ligne le 01 décembre 2015, consulté le 01 mai 2019. URL : http://journals.openedition.org/edc/6465 ; DOI : 10.4000/ edc. 6465 


\section{Quand le numérique s'invite au château: les serious games comme outil de médiation du patrimoine}

When digital technology storms the castle: serious games as a tool reflexion on heritage 
Résumé / Abstract

Dans cet article, nous examinons comment quatorze lieux de patrimoine ont recours à des serious games comme outil de médiation culturelle. Notre modèle d'analyse en trois temps (données, processus, aboutissement) se penche sur les types de contenus dispensés, sur les caractéristiques des jeux ainsi que sur leurs objectifs cognitifs, affectifs et communicatifs.

Mots-clés : serious game, patrimoine, médiation culturelle, éducation.
In this article, we examine the way in which fourteen heritage sites use serious games as a tool for cultural education. Our three-step analysis model (input, process and outcome) addresses the types of content offered, the characteristics of the games and their cognitive, emotional and communicative aims.

Keywords: serious game, cultural heritage, cultural education. 
L'envol du numérique, en particulier le numérique mobile, n'est aujourd'hui un secret pour personne. En 2014, davantage de personnes (46\%) ont un smartphone qu'un téléphone portable (43\%) et $29 \%$ des Français ont une tablette, qui se vend davantage qu'un ordinateur (Bigot, Croutte, 2014, 58-98). Compte tenu de l'intégration de plus en plus importante des supports numériques dans notre vie quotidienne, on mesure sans peine le potentiel du numérique au sein des institutions culturelles, comme outil de communication ou d'aide à la visite. Ce potentiel est d'ailleurs déjà particulièrement exploité, comme en témoignent les 325 applications mobiles muséales françaises recensées par le Club Innovation \& Culture France au 15 janvier 2015.

Dans le cadre de cet article, nous nous pencherons plus particulièrement sur les serious games, autrement dit sur les jeux vidéo à finalité éducative, auxquels on peut jouer au moyen de l'ordinateur ou de la tablette. L'objet de notre article concerne plus précisément les modalités de sélection et de représentation du patrimoine dans quinze serious games créés pour des lieux de patrimoine matériel (châteaux, abbaye, cathédrale, etc.) situés en France, au Royaume-Uni et en Belgique. L'enjeu de l'article consiste à déterminer quelles approches du patrimoine ces jeux favorisent, et au moyen de quels types d'activités.

Compte tenu de la nature différente des visites, nous avons choisi de nous limiter aux lieux qui se visitent pour eux-mêmes et non essentiellement pour les collections qu'ils abritent; c'est pourquoi nous avons exclu de notre corpus les jeux proposés par les musées, comme le jeu Sauvons le Louvre (2014) par exemple.

Notre article est composé de trois parties. Nous commencerons par présenter notre modèle d'analyse des serious games. La deuxième partie sera consacrée aux jeux composant notre corpus, que nous analyserons dans la troisième et dernière partie.

\section{1 \\ Un modèle d'analyse des serious games}

Formalisé pour la première fois en 1970 dans l'ouvrage Serious Games de Clark Abt, qui voyait dans ces jeux la possibilité de combiner des apprentissages scolaires et informels, le concept de serious game est aujourd'hui largement utilisé, tant par les chercheurs que par les industries culturelles. Si les définitions existantes se distinguent dans leur approche plus ou moins large de cette notion, elles se rejoignent dans leur insistance sur les finalités plurielles de ces jeux, qui dépassent le divertissement. Julian Alvarez, Damien Djaouti et Olivier Rampnoux $(2011,50)$ le définissent de la façon suivante: «application informatique, dont l'intention initiale est de combiner, avec cohérence, à la fois des aspects sérieux (serious) tels, de manière non exhaustive et non exclusive, 
l'enseignement, l'apprentissage, la communication, ou encore l'information, avec des ressorts ludiques issus du jeu vidéo (game)». Cette définition souligne particulièrement la diversité des finalités sérieuses possibles, qui ne se limitent pas à l'apprentissage stricto sensu. L'analyse des jeux révèlera en effet que de nombreux jeux visent surtout à sensibiliser les joueurs découvrant le patrimoine ou à leur donner envie de visiter, avant d'enseigner un contenu précis.

Afin d'identifier les types de contenus et les modalités d'apprentissage privilégiés par ces jeux, nous avons construit le modèle d'analyse ci-dessous ${ }^{1}$, en nous basant particulièrement sur certaines théories relevant des games studies (Garris et al., 2002, Mitgutsch et Alvarado, 2012), des sciences cognitives (Krathwohl 2002, Wouters et al., 2009) et des sciences de l'éducation (de Ketele et Roegiers, 2001). En complément de l'analyse des jeux au moyen de ce modèle, nous avons pour chaque jeu pris contact avec une personne qui a pris part à sa conception, afin de prendre connaissance des circonstances de sa création et des objectifs poursuivis par l'institution commanditaire ${ }^{2}$.

Le modèle prend en compte deux dimensions des objets ludiques étudiés: les données qui précèdent la partie renvoient au contenu à enseigner et aux caractéristiques ludiques du serious game; l'aboutissement concerne les objectifs escomptés par le concepteur.

\subsection{Le contenu à enseigner}

La taxonomie de Benjamin Bloom révisée par David Krathwohl (2002) nous permet d'identifier les types de connaissance mobilisés dans les serious games. Elle prend en compte, du plus simple au plus complexe: 1) les connaissances factuelles, qui constituent le socle de base sur lequel une matière peut être apprise. Cela concerne la terminologie et les éléments de base spécifiques à une matière; 2) les connaissances conceptuelles, qui croisent les éléments de base dans une structure plus large. Elles comprennent les classifications et catégories, les principes et généralisations, et les théories, modèles et structures; 3) les connaissances procédurales, qui renvoient au faire: compétences, techniques et méthodes, choix des procédures à appliquer; 4) les connaissances métacognitives concernent la connaissance de la cognition en général mais permettent également de prendre conscience de son propre fonctionnement cognitif.

1 Notre modèle n'est pas exhaustif ; nous nous sommes ici limitée aux dimensions que nous mobilisons pour cette étude. Le modèle de Garris et al. prend en compte la pratique empirique du joueur dans une troisième dimension. L'aboutissement peut lui aussi être abordé de façon empirique, ce qui n'est pas l'objet de cet article.

2 Les personnes avec qui nous avons pu échanger sont indiquées et remerciées à la fin de l'article. 


\subsection{Les caractéristiques du jeu}

Les quatre premiers axes d'analyse sont communs à tous les jeux vidéo, qu'ils soient de nature purement divertissante ou non. Le premier axe concerne I'univers fictionnel: Comprend-il une intrigue? Dans l'affirmative, est-elle linéaire, arborescente, ouverte, fermée, etc.? Le deuxième axe renvoie à la mécanique du jeu: quelles sont les règles? Quels sont les buts du jeu? Quels systèmes de récompenses et de feedback le jeu propose-t-il au joueur? Les stimuli sensoriels sont pris en compte dans le troisième axe: les signes visuels et sonores sont-ils soignés? Participent-ils à rendre l'expérience de jeu agréable? Enfin, le contrôle du jeu relève du quatrième axe, par lequel on examine dans quelle mesure la progression du jeu est contrôlée par le joueur ou par l'interface.

Le cinquième axe, spécifique aux serious games, porte sur l'accès au contenu. II se penche sur les types d'activités d'apprentissage proposées au joueur. Sur base de la typologie des activités d'apprentissage élaborée par Jean-Marie de Ketele et Xavier Roegiers (2001, 176-189), nous distinguons les quatre types d'activité suivants: les activités d'exploration, destinées à stimuler un nouvel apprentissage avant tout traitement proprement dit d'un contenu à enseigner; les activités d'apprentissage systématique qui permettent de fixer les savoirs et de systématiser les savoir-faire, éventuellement découverts lors d'activités d'exploration; les activités de structuration, visent, comme leur nom l'indique, à structurer les savoirs et savoir-faire et les activités d'intégration stimulent les liens entre les différents savoirs et savoir-faire et intègrent ceux-ci dans des activités qui les mobilisent conjointement.

\subsection{L'aboutissement (objectifs visés par les concepteurs)}

Neuf objectifs peuvent être visés par les concepteurs d'un serious game, regroupés en objectifs cognitifs, affectifs et communicatifs. Les six premiers relèvent de la taxonomie des apprentissages cognitifs élaborée par Bloom et révisée par Krathwohl (2002): mémoriser (reconnaître et garder en mémoire), comprendre (interpréter), appliquer (exécuter, implémenter), analyser, évaluer, créer (générer).

Nous avons complété les objectifs cognitifs par les concepts d'objectifs affectifs et communicatifs étudiés par Pieter Wouters et al. (2009). Les objectifs affectifs concernent ici les changements d'attitudes et des représentations d'un sujet donné, ainsi que la motivation à poursuivre son apprentissage du sujet, grâce aux procédés de sensibilisation. Enfin, si la plupart des jeux se jouent de façon individuelle, certains peuvent développer une dimension collective, et ainsi développer des objectifs communicatifs. 
Le schéma ci-dessous récapitule notre modèle.

DONNEES

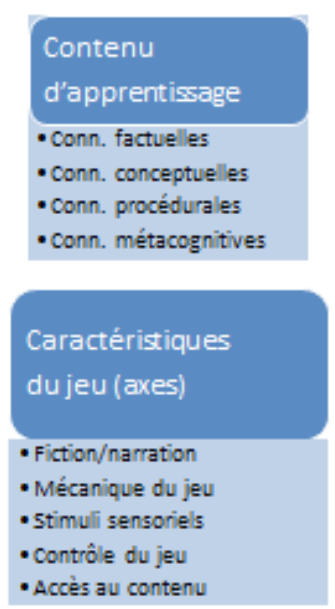

ABOUTISSEMENT

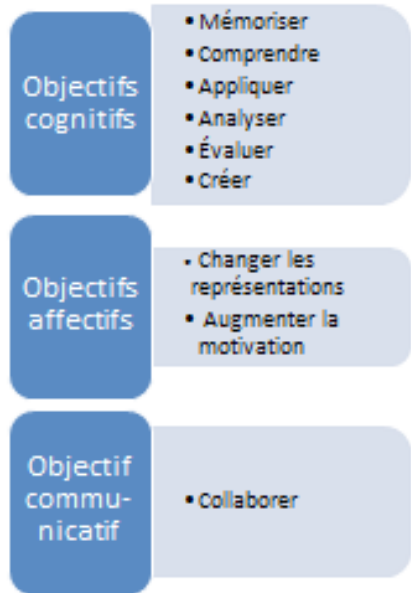

Tableau 1: Notre modèle d'analyse des serious games basé sur de Ketele et Roegiers (2001), Garris et al. (2002), Krathwohl (2002), Mitgutsch et Alvarado (2012) et Wouters et al. (2009)

\section{2 \\ Le patrimoine matériel en quinze jeux}

Avant d'appliquer ce modèle d'analyse à notre corpus, présentons-le plus en détails. Celui-ci comprend quinze jeux, dont la variété témoigne des multiples possibilités ludiques: ils s'adressent aux enfants, aux adultes ou à toute la famille; ils se jouent à la maison ou sur tablette lors de la visite, relèvent de différents genres vidéoludiques et durent de quelques minutes (Les Grottes de Gargas, Slye's lost words, Become a luthe hero) à une quarantaine d'heures (Ofabulis). Nous avons choisi de prendre en compte la totalité des jeux proposés par les lieux de patrimoine que nous avons identifiés, de façon à pouvoir dégager les premières tendances de cette pratique en plein développement ${ }^{3}$. Le corpus se veut donc le plus étendu possible, sans pour autant prétendre à l'exhaustivité puisqu'il est possible que nous n'ayons pas pris connaissance de l'existence de certains jeux. Le corpus a été constitué en consultant les moteurs de recherche et les bases de données consacrées aux serious games et/ou aux applications numériques ainsi qu'en interrogeant plusieurs experts des serious games. Le tableau suivant présente le corpus:

3 Les sites Internet utilisés sont http://www.club-innovation-culture.fr/, http:// serious.gameclassification.com/FR/ et http://www.seriousgame.be/. Seul le jeu Complot à la Corderie (2012) proposé par la Corderie Royale de Rochefort n'a pas pu être testé car il s'accompagne d'une mise en fiction sur le site uniquement proposée en été. 


\begin{tabular}{|c|c|c|c|c|}
\hline $\begin{array}{l}\text { Nom du jeu } \\
\text { (année) }\end{array}$ & $\begin{array}{l}\text { Patrimoine } \\
\text { (pays) }\end{array}$ & Public cible & $\begin{array}{l}\text { Expérience } \\
\text { ludique }\end{array}$ & $\begin{array}{c}\text { Cenre } \\
\text { vidéoludique }\end{array}$ \\
\hline $\begin{array}{l}\text { Les Grottes de } \\
\text { Gargas (2006) }\end{array}$ & $\begin{array}{l}\text { Les Grottes de } \\
\text { Gargas (F) }\end{array}$ & Famille & $\begin{array}{l}\text { Ordinateur } \\
\text { à la maison } \\
\text { et sur site }\end{array}$ & Simulation \\
\hline $\begin{array}{l}\text { Slye's lost words } \\
\text { (2013) }\end{array}$ & $\begin{array}{l}\text { Théâtre du Globe } \\
\qquad(\mathrm{R}-\mathrm{U})\end{array}$ & 5-11 ans & $\begin{array}{l}\text { Ordinateur } \\
\text { à la maison }\end{array}$ & Quiz-puzzle \\
\hline $\begin{array}{l}\text { Hemming's (2013) } \\
\text { play company }\end{array}$ & $\begin{array}{l}\text { Théâtre du Globe } \\
\qquad(\mathrm{R}-\mathrm{U})\end{array}$ & 5-11 ans & $\begin{array}{l}\text { Ordinateur } \\
\text { à la maison }\end{array}$ & Simulation \\
\hline $\begin{array}{c}\text { Become a Luthe } \\
\text { hero (2013) }\end{array}$ & $\begin{array}{l}\text { Théâtre du Globe } \\
\qquad(R-U)\end{array}$ & 5-11 ans & $\begin{array}{l}\text { Ordinateur } \\
\text { à la maison }\end{array}$ & Simulation \\
\hline $\begin{array}{c}\text { Walk Through a } \\
\text { Victorian House } \\
\text { (2008) }\end{array}$ & $\begin{array}{l}\text { Geffrye Museum } \\
(R-U)\end{array}$ & $\begin{array}{c}\text { Non } \\
\text { mentionné } \\
( \pm 8-12 \text { ans })\end{array}$ & $\begin{array}{l}\text { Ordinateur } \\
\text { à la maison }\end{array}$ & Simulation \\
\hline Fast Car (2011) & $\begin{array}{l}\text { Patrimoine } \\
\text { de l'Unesco } \\
\text { (5 continents) }\end{array}$ & $\begin{array}{l}\text { Adolescents } \\
\text { et adultes }\end{array}$ & $\begin{array}{l}\text { Ordinateur } \\
\text { à la maison }\end{array}$ & Course de voitures \\
\hline $\begin{array}{l}\text { Unlock the dragon } \\
\text { (2012) }\end{array}$ & $\begin{array}{l}\text { Tour de Londres } \\
\text { (R-U) }\end{array}$ & $\begin{array}{l}\text { Non } \\
\text { mentionné } \\
\text { (adolescents } \\
\text { et adultes) }\end{array}$ & $\begin{array}{l}\text { Ordinateur } \\
\text { à la maison }\end{array}$ & Quizz-puzzle \\
\hline $\begin{array}{l}\text { Meet the royal } \\
\text { beasts (2012) }\end{array}$ & $\begin{array}{l}\text { Tour de Londres } \\
(R-U)\end{array}$ & $\begin{array}{l}\text { Non } \\
\text { mentionné } \\
\text { (adolescents } \\
\text { et adultes) }\end{array}$ & $\begin{array}{l}\text { Ordinateur } \\
\text { à la maison }\end{array}$ & Quizz-puzzle \\
\hline $\begin{array}{l}\text { Les Bozzons } \\
\text { (2012) }\end{array}$ & $\begin{array}{c}\text { Abbaye } \\
\text { de Fontevraud (F) }\end{array}$ & $9-14$ ans & Tablette sur site & Aventure \\
\hline $\begin{array}{c}\text { Pagaille à } \\
\text { Versailles (2012) }\end{array}$ & $\begin{array}{c}\text { Château } \\
\text { de Versailles (F) }\end{array}$ & $8-12$ ans & $\begin{array}{l}\text { Ordinateur } \\
\text { à la maison }\end{array}$ & Simulation \\
\hline $\begin{array}{c}\text { Enigmes à } \\
\text { Versailles (2013) }\end{array}$ & $\begin{array}{c}\text { Château } \\
\text { de Versailles (F) }\end{array}$ & $\begin{array}{l}\text { Adolescents } \\
\text { et adultes }\end{array}$ & Tablette sur site & Aventure \\
\hline $\begin{array}{l}\text { Chalucet et les } \\
\text { explorateurs du } \\
\text { temps (2013) }\end{array}$ & $\begin{array}{l}\text { Château de } \\
\text { Chalucet (F) }\end{array}$ & Famille & $\begin{array}{c}\text { Ordinateur } \\
\text { à la maison } \\
\text { et } 2^{\mathrm{e}} \text { partie } \\
\text { sur tablette sur site }\end{array}$ & Aventure \\
\hline Ofabulis (2014) & $\begin{array}{c}19 \text { monuments } \\
\text { du Centre } \\
\text { des monuments } \\
\text { nationaux }\end{array}$ & $\begin{array}{l}\text { Adolescents } \\
\text { et adultes }\end{array}$ & $\begin{array}{l}\text { Ordinateur } \\
\text { à la maison }\end{array}$ & Aventure \\
\hline $\begin{array}{l}\text { Le Livre des } \\
\text { siècles (2014) }\end{array}$ & $\begin{array}{c}\text { Château de } \\
\text { Fontainebleau (F) }\end{array}$ & 8-13 ans & Tablette sur site & Aventure \\
\hline $\begin{array}{c}\text { Hypergothique, } \\
\text { le secret des } \\
\text { bâtisseurs (2015) }\end{array}$ & $\begin{array}{c}\text { Collégiale Sainte- } \\
\text { Waudru (B) }\end{array}$ & $\begin{array}{l}\text { Adolescents } \\
\text { et adultes }\end{array}$ & Tablette sur site & Aventure \\
\hline
\end{tabular}




\section{Analyse des serious games}

Examinons les tendances qui se dégagent de l'analyse de ces quinze serious games du point de vue du contenu patrimonial éprouvé, des caractéristiques principales du jeu et des objectifs attendus par les concepteurs.

\subsection{Le contenu patrimonial proposé au joueur}

Trois tendances en matière de contenu proposé peuvent être observées. Premièrement, tous les jeux fournissent quelques informations factuelles (dates, noms, etc.) mais privilégient une approche qui articule différentes informations qui, ensemble, permettent de comprendre le fonctionnement des lieux et le sens des espaces, au fil des siècles. Les mini-jeux Les Grottes de Gargas, Become a luthe hero et Slye's lost words comprennent très peu de contenu, ce qui peut s'expliquer par la taille réduite du jeu.

Deuxièmement, trois jeux activent des connaissances procédurales en plaçant le joueur dans la peau d'un expert: un archéologue qui découvre les traces des dessins dans Les Grottes..., un historien dans Chalucet..., un directeur de compagnie théâtrale dans Hemming's... Excepté pour ce dernier exemple, ces connaissances procédurales ne constituent toutefois pas la finalité de l'apprentissage mais permettent une approche ludique du contenu, par manipulation virtuelle des éléments du patrimoine.

Troisièmement, les jeux visent particulièrement un contenu anecdotique, qui singularise le lieu (la présence d'un groupe de faussaires dans le château de Chalucet au XVe siècle, par ex.) ou qui surprend nos représentations (les moinesses de Fontevraud dormaient toutes habillées, par ex.). Nos représentations contemporaines sont parfois intégrées aux jeux, surtout destinés aux enfants, comme dans les affirmations de l'avatar Ruby qui visite la maison victorienne et qui s'étonne que tout y est si démodé (Walk Through A Victorian House). Dans une même optique de différenciation, sept jeux vont mettre en évidence la singularité des lieux en mettant en scène des légendes attachées au lieu (Ofabulis, Chalucet et les explorateurs du temps, Hypergothique ou le secret des bâtisseurs, Unlock the Dragon ${ }^{4}$ ) ou des secrets fictifs (secret du Livre des siècles, secret de la présence des bozzons à Fontevraud) ou authentiques (secret de Visconti dans Enigmes à Versailles).

Les serious games du corpus ont donc en commun de proposer un contenu limité et humanisé (à hauteur d'homme) du lieu, pensé en complément d'une visite classique. Deux jeux font exception dans cette tendance. Alors que tous

4 Dans ce cas, les légendes sont mentionnées mais ne font pas l'objet d'une mise en scène. 
les jeux ciblent un joueur encore peu informé sur le patrimoine en question, Enigmes... vise les abonnés du château de Versailles, familiarisés avec son histoire et en attente d'informations complémentaires. Il ne comprend donc pas les informations de base sur le château mais guide directement le joueur dans les légendes de Diane et d'Apollon, en la compagnie de Visconti. Hypergothique... se démarque quant à lui des autres jeux par l'ampleur du contenu offert, très pointu et relativement complet, tant sur le lieu que sur l'architecture gothique en général.

\subsection{Les caractéristiques des jeux}

Dans le cadre de cet article, nous nous concentrerons ici sur deux axes du modèle, portant respectivement sur l'univers fictionnel (l'intrigue développée) et sur l'accès au contenu patrimonial. Les autres caractéristiques des jeux (mécanique du jeu, stimuli, contrôle) pourront faire l'objet d'une analyse spécifique ultérieure.

Typique des jeux d'aventure, l'univers fictionnel se tisse dans une intrigue dans les cinq jeux d'aventure sur les six qui composent notre corpus; seul le jeu Les Bozzons n'est pas organisé autour d'une intrigue. Ces jeux comprennent une seule intrigue, sous la forme d'une quête: retrouver les plans de la collégiale (Hypergothique...) ou un livre perdus (Le Livre des siècles), sauver le château des ténèbres qui le menacent (Enigmes...) et aider l'héroïne à se situer dans le temps (Chalucet...). Les différentes épreuves permettent de récolter des indices qui mèneront à l'objet de la quête. Ofabulis se démarque par le nombre de quêtes proposées: pas moins de cinquante quêtes permettent de récolter des indices qui mènent à la résolution de l'intrigue.

Les jeux d'exploration des espaces obéissent le plus souvent à une organisation linéaire, qui n'offre pas de personnalisation du parcours (Les Bozzons, Fast Car). Dans Enigmes... et Le Livre des siècles, l'ordre des étapes du parcours correspond au parcours lui-même linéaire d'une visite classique, qui ne permet pas de faire demi-tour entre les salles des châteaux. Le jeu Pagaille... suit la chronologie de la construction du château et est donc linéaire mais il propose de choisir l'ordre des épreuves rassemblées sous la même décennie de construction. Pour Hypergothique..., l'organisation spatiale de la collégiale Sainte-Waudru, qui comprend un seul grand espace, permet un parcours pour lequel on peut choisir l'ordre des huit stations, même si leur numérotation invite à les découvrir dans un ordre préétabli. De même pour Walk... et Chalucet..., le jeu sur ordinateur permet de choisir l'ordre d'exploration des différentes zones du lieu. Plus le jeu est long, plus il complexifie et enrichit les rapports entre les différentes épreuves: Ofabulis débute par un parcours linéaire puis propose différents embranchements aux joueurs, qui disposent ainsi d'un parcours plus personnalisé. Ofabulis est également le seul jeu qui ne fournit pas in 
fine le même contenu à tous les joueurs, en fonction des choix qu'ils opèrent ${ }^{5}$.

Les cinq jeux qui développent une intrigue sont aussi ceux qui invitent le plus à explorer les lieux, virtuellement ou lors d'une véritable visite et ne comprennent pas de timing; le joueur ne doit donc pas se battre contre le temps pour gagner. Les autres jeux comprennent tous des timings pour les épreuves au sein du jeu mais pas de timing général pour le jeu dans sa totalité; entre les épreuves, le joueur peut toujours mettre le jeu en pause et le poursuivre à son rythme.

Examinons à présent comment l'accès au contenu patrimonial est pensé. Quatre grands cas de figures peuvent être observés:

1. Pas de contenu fourni avant ou après le jeu: le jeu est introduit par un nombre très limité d'informations; le joueur est invité à jouer directement et l'apprentissage se fait uniquement par le jeu (Les Grottes..., Become..., Hemming's...);

2. Le contenu précède l'épreuve ludique:

a. Contenu (obtenu directement ou en cliquant sur des éléments particuliers) puis question sur ce contenu,

b. Contenu puis concrétisation de ce contenu dans une épreuve ludique ;

3. L'épreuve ludique précède le contenu :

a. Question à choix multiple puis explication,

b. Épreuve ludique basée sur l'exploration ou la manipulation de détails du lieu puis explication;

4. Seul le contexte lie le contenu et l'épreuve ludique.

Les cas $n^{\circ} 1$ et 3 comprennent des activités d'exploration, tandis que le cas $n^{\circ} 2$ relève davantage d'activités d'apprentissage systématique. Les jeux de notre corpus ne comprennent pas d'activités de structuration ou d'intégration, plus complexes que les deux premiers types, portant eux sur des apprentissages plus isolés les uns des autres. L'apprentissage est donc plus ponctuel qu'intégratif. Notons qu'aucun jeu ne réactive des connaissances apprises quelques instants auparavant; tous ne manipulent le contenu qu'une seule fois.

Notons que plusieurs jeux proposent du contenu supplémentaire facultatif (Pagaille..., Le Livre des siècles) ou qui nous est proposé de passer si on le souhaite (Ofabulis, Hypergothique...).

Détaillons les articulations entre la connaissance et le jeu ci-après. Le cas $n^{\circ} 1 n^{\prime} e s t$ pas détaillé puisque le jeu ne dispense presque pas de contenu. 


\section{Cas $n^{\circ} 2$ : le contenu précède l'épreuve ludique}

Trois jeux posent des questions au joueur après lui avoir fourni l'information. Walk... et Les Bozzons vérifient la compréhension des textes en posant une question à choix multiple après leur lecture. Ofabulis pose des questions à la fin de l'épreuve de plusieurs minutes et non directement après la présentation du contenu.

Quatre jeux concrétisent le contenu dans un défi ludique. Les Bozzons (après la question), Meet... et Pagaille... concrétisent les quelques informations obtenus dans des activités ludiques qui les mettent en action: par exemple, Meet... nous apprend qu'au XVIII e siècle les visiteurs de la Tour de Londres lançaient des clous aux autruches, pensant qu'elles pouvaient digérer le métal. Après cette information, le jeu consiste à bien placer l'autruche de façon à ce qu'elle avale le plus de clous possible dans un temps imparti. Dans le même ordre d'idées, Pagaille... explique rapidement les jardins de Versailles puis propose au joueur de semer les bonnes graines aux bons endroits. Enfin, Hypergothique... propose de dessiner sur la tablette les traces-signatures laissées par les maîtres de carrière dans les colonnes en pierre de la collégiale.

Cas $n^{\circ} 3$ : l'épreuve ludique précède le contenu

À l'inverse des jeux relevant du deuxième cas, cinq jeux proposent au joueur de commencer par jouer puis de recevoir quelques explications. Dans les cas de Fast Car et d'Unlock..., le joueur doit répondre aux questions sans connaissance préalable, puis bénéficie d'informations, que sa réponse soit juste ou non.

«Tu ne vois pas, apprends à regarder autour de toi, lève la tête!», indique le personnage du Livre des siècles au joueur. Trois jeux (Enigmes..., Le Livre des siècles et Chalucet...) proposent un jeu basé sur l'exploration ou la manipulation virtuelle d'éléments du lieu. Dans un deuxième temps, quelques informations sont fournies: dans Enigmes..., le joueur doit par exemple identifier auquel des quatre éléments (eau, terre, air, feu) les fragments d'un plafond font référence ; le joueur du Livre des siècles doit retrouver les sept erreurs qui se sont glissées dans une fresque; celui de Chalucet... doit quant à lui reconstituer le sol carrelé d'une salle du château, etc.

Cas $n^{\circ} 4$ : seul le contexte lie le contenu et l'épreuve ludique

Dans ce rare cas, le jeu mobilise le lieu patrimonial uniquement comme contexte mais est indépendant de l'apprentissage du contenu donné. Dans Hypergothique... par exemple, après avoir reçu des informations sur la châsse de la collégiale, le jeu consiste à faire descendre virtuellement la châsse sans la faire tomber, grâce à des cordes à gauche et à droite.

Il apparaît que les cas $n^{\circ} 2$ (sept jeux) et $n^{\circ} 3$ (cinq jeux) sont les plus fréquents: le contenu et l'épreuve ludique s'articulent étroitement, l'un précédant l'autre (cas $n^{\circ} 2$ ) ou lui succédant (cas $n^{\circ} 3$ ). Une grande tendance ne se démarque donc pas au sein de notre corpus. 


\subsection{L'aboutissement (objectifs visés par les concepteurs)}

Nous avions identifié neuf objectifs escomptés par les concepteurs de serious games: mémoriser, comprendre, appliquer, analyser, évaluer, créer, changer les représentations, augmenter la motivation et collaborer. Sept objectifs sur les neuf qui composent notre modèle sont observables au sein de notre corpus; les objectifs d'évaluation et de création nous semblent absents des jeux de notre corpus.

Aucun jeu ne vise à mémoriser au sens de garder en mémoire des faits précis ou des dates. La mémorisation est plutôt visuelle: les jeux permettent une première expérience visuelle du lieu, que ce soit chez soi ou sur site. Notons que Chalucet... est le seul jeu qui n'offre pas de représentation visuelle du château: au terme de la partie jouée à la maison, le joueur ne sait pas encore à quoi il ressemble.

Plutôt que la mémorisation de faits précis, c'est la fonction et le sens des espaces que le joueur est invité à comprendre grâce au jeu. Certains jeux vont viser cette compréhension en aiguisant particulièrement le regard du joueur, en l'invitant à observer les détails pour comprendre le sens des espaces. Analyser consiste à être capable d'organiser les différentes parties d'un tout et ainsi de les mettre en perspective. On peut considérer cet objectif comme le prolongement de l'objectif de comprendre: I'information n'est pas seulement traitée, elle est organisée au sein d'un système global qui fait sens. Comprendre et analyser sont des objectifs centraux pour la visite d'un lieu de patrimoine; celle-ci fait découvrir au joueur la vie au cœur de ces espaces vieux de plusieurs siècles. Le joueur peut ainsi en saisir le sens pièce par pièce et au niveau du lieu patrimonial dans son ensemble.

L'objectif d'appliquer se traduit par une mise en pratique ludique, en se mettant dans la peau d'un archéologue (Chalucet...) d'un assistant du roi ( $\mathrm{Pa}$ gaille...) ou d'un scientifique (Les Grottes...). II ne s'agit pas d'application pointue mais plutôt d'une mise en situation concrète.

Les deux objectifs affectifs (changer les représentations et augmenter la motivation) sont particulièrement importants pour les jeux. De nombreux jeux visent en effet à rompre les représentations négatives (surtout des enfants) envers le lieu patrimonial en leur offrant une expérience plaisante et motivante. Halia Smail, en charge de la médiation des publics à l'abbaye de Fontevraud', soulignait les représentations positives dont pouvait jouir un château auprès des enfants et dont une abbaye était dépourvue. Rendre la visite de ces lieux agréable pour les enfants est un enjeu à part entière pour Les Bozzons, et plus généralement pour les jeux destinés aux enfants. Dans un autre ordre d'idées, Enigmes... vise lui aussi à surprendre le public d'abonnés, en lui offrant un 
contenu sur Versailles qui sort des sentiers battus. Ofabulis stimule quant à lui l'attention active et les réflexes d'observation des détails et de recherche d'informations de ses joueurs. Ce jeu est destiné à des "gamers», autrement dit à des joueurs réguliers de jeux vidéo, qui ont l'habitude de résoudre des énigmes virtuelles. Au-delà d'un réel apprentissage sur les lieux de patrimoine, Ofabulis espère qu'ils transposeront leurs réflexes d'observation et de recherche d'informations à d'autres contextes de découverte culturelle, notamment lors de visites de sites.

Enfin, ce jeu comprend un objectif plus rare, celui de stimuler la collaboration entre les joueurs. Dans Ofabulis, il est nécessaire de collaborer avec d'autres joueurs en réseau pour résoudre l'intrigue, car chaque profil de joueur dispose d'informations spécifiques, à partager avec les joueurs aux autres profils. Chalucet... stimule lui aussi les collaborations dans la mesure où ce jeu nécessite la participation d'un adulte; les épreuves de niveaux variés font intervenir chaque membre de la famille.

\section{4 \\ Conclusion}

La plupart des jeux se rejoignent dans leur prédilection pour un contenu conceptuel, dont l'objectif consiste dans une mémorisation visuelle et une compréhension des espaces, via des activités d'exploration (surtout quand le contenu précède le jeu) ou d'apprentissage systématique ludique (quand il lui succède). L'équation entre divertissement et apprentissage dépend également du public visé (visiteur d'un jour ou habitué, amateur de jeux ou pas, etc.), de la notoriété établie du lieu - les enjeux sont naturellement différents pour Versailles ou un château en Haute-Vienne...- - ou des outils de médiation déjà à disposition des visiteurs. Concernant ce dernier point, il apparaît important pour les lieux de patrimoine d'offrir les jeux sur tablette à utiliser sur site en complémentarité par rapport aux visites guidées. Le serious game se doit de proposer un nouvel angle de visite, voire un contenu alternatif (comme dans le cas d'Enigmes...) qu'il importe de ne pas mettre en concurrence avec la visite classique. Le cas de Versailles nous montre également que le positionnement alternatif $\mathrm{du}$ serious game peut aussi s'expliquer d'un point de vue institutionnel: visiter un tel haut lieu de l'Histoire de France sur base d'un jeu sort encore des sentiers battus et peut quelque peu entrer en contradiction avec la majesté des lieux ${ }^{7}$.

7 Lors d'un entretien réalisé le 24 février 2015, Mathias Le Galic, chef du service des programmes culturels et scolaires du château de Versailles et concepteur du jeu Enigmes..., soulignait la divergence des points de vue institutionnels possibles entre l'équipe de conservation du patrimoine et celle de la médiation des publics, plus encline à utiliser les nouvelles technologies. 
Enfin, il apparaît que si certains jeux tissent des rapports étroits entre divertissement et apprentissage, et ainsi entre une participation passive et une participation plus active qui permet de réellement apprendre en jouant, d'autres construisent des relations parfois distendues entre ces deux pôles. Ils alternent des activités d'apprentissage et des épreuves divertissantes et misent souvent davantage sur l'apprentissage, le jeu servant d'intermède récréatif. De cette façon, ces jeux n'exploitent pas tout le potentiel intégratif des jeux vidéo comme outil d'apprentissage culturel.

Notons pour terminer que si les visites guidées s'adressent à tous les visiteurs, les serious game semblent davantage devoir cibler une partie de leur public, les réactions envers le jeu, enthousiastes ou réfractaires, montrant qu'il est difficile de contenter tout le monde. Pour Edwige Lelièvre ${ }^{8}$, conceptrice d'Ofabulis et des Mystères de la Basilique, créer un jeu sans cible précise est d'ailleurs une gageure, tant les différences de niveau, d'âge, de connaissances culturelles, etc., impliquent des attentes spécifiques. L'un des enjeux consiste donc à éviter le grand écart qui viserait le plus grand nombre mais qui risquerait in fine à dénaturer l'expérience culturelle proposée.

\section{Remerciements}

Je remercie vivement les personnes mentionnées ci-dessous pour leur gentillesse et leur disponibilité lors de nos entretiens

David De Oliveira, business developer chez FuretCompany, société qui a créé la conception technique d'Enigmes à Versailles et de Chalucet et les explorateurs du temps.

Mathieu Dumenil, chef du projet Hypergothique et le secret des bâtisseurs.

Mathias Le Galic, chef du service des programmes culturels et scolaires du château de Versailles et concepteur du jeu Enigmes à Versailles.

Edwige Lelièvre, conceptrice d'Ofabulis et des Mystères de la Basilique, maître de conférences à l'Université de Versailles Saint-Quentin-en-Yvelines.

David Millerou, chef du département éducatif du château de Fontainebleau.

Frédéric Rivet, dirigeant de la société Minuit Moins Dix, qui a réalisé Chalucet et les explorateurs du temps avec la société FuretCompany.

Halia Smail, en charge de la médiation des publics à l'abbaye de Fontevraud. 
Bibliographie

Abt C.C. (1970). Serious games,

New York, Viking Press, $196 \mathrm{p}$.

Alvarez J., DjaoutiD., Rampnoux O. (2011). "Typologie des serious games». In RufatS. et Ter Minassian H. (dir.), Les Jeux vidéo comme objet de recherche, Paris, Questions Théoriques, p.46-65.

Bigot R., CroutteP. (2014). La Diffusion des technologies de l'information et de la communication dans la société française, Étude réalisée à la demande du Conseil Général de l'Économie, de I'Industrie, de l'Énergie et des Technologies (CGE) et de l'Autorité de Régulation des Communications Électroniques et des Postes (ARCEP), CREDOC, $\mathrm{n}^{\circ} 317$.

Garris R., Ahlers R., Driskell J.E. (2002). "Games, motivation, and learning: a research and practice model ». In Simulation \& Gaming, Vol.33, n 4 , p. 441-467.

KrathwohID.R. (2002). «A Revision of Bloom's taxonomy: an overview ". In Theory into Practice, Vol.41, $n^{\circ} 4$, p. 212-218.

Mitgutsch K., AlvaradoN. (2012). «Purposeful by design?: a serious game design assessment framework ". In Actes de I'International Conference on the Foundations of Digital Games, New York, ACM, p.121-128.
Roegiers X., De Ketele J.-M. (2001).

Une pédagogie de l'intégration.

Compétences et intégration

des acquis dans

l'enseignement, Bruxelles, De Boeck.

Wouters P., Van der SpekE.D., Van

OostendorpH. (2009). "Current

Practices in Serious Game Research:

A Review from a Learning

Outcomes Perspective». In

Connolly T., Stansfield M.

et BoyleL. (dir.), Current Practices

in Serious Game Research:

A Review from a Learning

Outcomes Perspective, Hershey, Information Science Reference. 
